\title{
Osteomyelitis Due to Citrobacter koseri Infection in a Diabetic Patient
}

\author{
Server Yagcia, ${ }^{\mathrm{a}}$, Kader Arslan ${ }^{\mathrm{a}}$, Zeliha Kocak Tufanª, Sami Kiniklia, Ali Pekcan Demiroz ${ }^{\mathrm{a}}$
}

\begin{abstract}
Citrobacter infections are reported in neonates and immunocompromised patients most commonly. Citrobacter koseri (diversus) is an important cause of neonatal meningitis and brain abscess formation. But osteomyelitis is a rare condition and very few cases were reported. We described a 68 -years-old man with diabetes mellitus who had 1st metatarsal osteomyelitis due to Citrobacter koseri. Intravenous treatment with piperacillin-tazobactame was administered for 3 weeks and the wound was recovered completely. Citrobacter koseri can be a cause of osteomyelitis in patients with underlying predisposing factors and bone cultures should be evaluated to detect probable pathogens and determine proper antimicrobial therapy.
\end{abstract}

Keywords: Citrobacter; Osteomyelitis; Diabetes

\section{Introduction}

Diabetic foot osteomyelitis is typically arises because of the development of skin ulcer due to vascular insufficiency, neuropathy and hyperglycemia. The diagnosis in these patients requires particular physical examination as a chronic ulcer with a surface larger than $2 \mathrm{~cm}^{2}$ associated with osteomyelitis as well as high levels of ESR and C-reactive protein (CRP) [1].

Citrobacter species are known to cause infections espe-

Manuscript accepted for publication June 9, 2011

${ }^{a}$ Ankara Training \& Research Hospital, Infectious Diseases \& Clinical Microbiology Department. Ulucanlar 06340 Ankara, Turkey

${ }^{\mathrm{b}}$ Corresponding author: Server Yagci.

Email: serveryagci@yahoo.com.tr

doi:10.4021/jem36e cially in neonates and immunocompromised hosts as well as nosocomial infections. Citrobacter koseri (diversus) has been found to cause meningitis and brain abscess besides sepsis in neonates $[2,3]$. Citrobacter osteomyelitis is a rare condition and there are only few septic arthritis and osteomyelitis cases reported due to Citrobacter species [4-6]. Here we report a case of $C$. koseri osteomyelitis in an elderly patient with diabetic foot infection. The concerned literature is reviewed and discussed.

\section{Case Report}

A 68-years-old man was referred to our outpatient department with purulent wound on his left-foot. His past medical history was significant for diabetes mellitus and he had been treated with oral anti-diabetic agents plus mixed insulin. He had also atherosclerotic vascular disease and was on low-molecular weight heparin therapy. The wound had been purulent for 3 months. He denied any kind of trauma to his foot or any fungal infection. Previously, the patient had referred to a physician for his wound and treatment of amoxicillin-clavulanate $1000 \mathrm{mg} 3$ x 1 PO was administered. Since the wound had worsened and purulence had continued, the patient was hospitalized. There was a necrosis and ulcerated area of $3 \times 3 \mathrm{~cm}$ on his left foot, around the 1st metatarsal bone. He was not febrile and remaining physical findings were normal. Remarkable laboratory findings were as follows: CRP; $0.837 \mathrm{mg} / \mathrm{dL}, \mathrm{ESR} ; 63 \mathrm{~mm} / \mathrm{h}$, Leukocytes; $5.2 \times 10^{3} / \mu \mathrm{L}$. Nuclear total body scintigraphy revealed $1 \mathrm{st}$ metatarsal osteomyelitis of the left foot. Aspiration culture from the purulence revealed Escherichia coli. Sulbactameampicillin 4 x 1.5 g plus ciprofloxacin 2 x $400 \mathrm{mg}$ IV therapies were administered. But no regression was recorded in the wound and it even worsened. Since the wound didn't heal bone cultures were taken for further investigation. Bone culture was obtained by surgical debridement and inoculated onto both aerobic and anaerobic agar media. Agar plates were incubated at $37^{\circ} \mathrm{C}$ in both aerobic and anaerobic conditions. After an incubation period of 24 hours a Gram negative bacterium was isolated. The bacterium was identified as C. koseri according to biochemical tests and confirmed by 
VITEK-2 Compact automated system (Biomerieux, France). It was resistant to ampicillin and the therapy was switched to piperacillin-tazobactame $3 \times 4.5 \mathrm{~g}$ IV according to the antibiotic susceptibility test results. Antibiotic treatment was administered for 3 weeks. The purulence decreased and the wound healed. Laboratory findings returned to normal levels. The patient was discharged to continue with peroral therapy with moxifloksasin 1 x $400 \mathrm{mg}$ for another three weeks and the total treatment was completed to 6 weeks. No relapse was seen on the follow-up.

\section{Discussion}

Citrobacter is aerobic, Gram-negative bacilli belonging to Enterobacteriaceae. C.amalonaticus, C. diversus (C. koseri ) and $C$. freundii are three major species in the genus. The most common sites that Citrobacter species cause infections are urinary tract, gastrointestinal tract, wound or decubitus and other sites respectively [2]. Citrobacter infections are usually found in immunocompromised hosts, old patients and neonates [7]. Neonatal Citrobacter infections are not associated with any particular underlying conditions. In contrast, in adults Citrobacter infections are seen especially in debilitated, immunosuppressed, or elderly patients. There are few reported cases of neonatal Citrobacter septic arthritis and osteomyelitis. Citrobacter osteomyelitis in the older patients often results from direct spread of a soft tissue infection because of vascular compromise such as diabetes mellitus, and bone cultures may yield mixed microorganisms $[5,6]$

Our patient is one of the very few reported cases of Citrobacter koseri (diversus) osteomyelitis. Osteomyelitis due to Citrobacter koseri has been reported in 2 elderly patients with diabetes mellitus only in one paper which is a retrospective study conducted in 1980, evaluating Citrobacter infections over a 6 year period. The clinical significance in these patients was indeterminate in one because of a small percentage of $C$. diversus in a culture that contained Staphylococcus aureus and Streptococcus agalactiae predominantly, and the other was termed secondary because of prior antimicrobial therapy in the patient. Citrobacter was one of the most common agents of osteomyelitis in their hospital and there was an underlying clinical condition that could have been the predisposing factor as trauma, neurosurgical surgery or diabetes mellitus [6]. Another case was a child with maxillary osteomyelitis, which led to meningitis by direct extension [8]. Citrobacter koseri osteomyelitis in our patient probably resulted from direct spread of the tissue infection because he had vascular disease and diabetes mellitus as predisposing factors besides his age.

As osteomyelitis is usually presents in association with an ulcer and soft tissue infection wide spectrum antibiotics should be initially given. Antibiotic selection is made ac- cording to the results of cultures. Bone cultures should be carried out but this is not always practical especially in ischemic feet [9]. Bone fragments in the base of the wound may be removed by debridement and then be sent for culture.

Because most of diabetic foot infections are polymicrobial, broad spectrum antibiotics should be used. It is useful to include antibiotics with good bone penetration such as sodium fusidate, rifampicin clindamycin and ciprofloxacin [10]. The best method for choosing proper antibiotics is microbiological examination of bone cultures in these patients. Initially E.coli was isolated from the aspiration culture of the purulence in our patient. Since the wound didn't heal even antimicrobial therapy was administered, bone culture was obtained and C. koseri was isolated. Soft tissue samples may reveal mixed pathogens predominantly in a diabetic foot specimen $[11,12]$. As a result it is crucial to obtain bone culture to detect probable pathogens in osteomyelitis resulting from diabetic foot infection.

Physicians should be aware that Citrobacter koseri can be a cause of osteomyelitis in debilitated, immunosuppressed, or elderly patients, and can respond favorably to proper antimicrobial therapy according to bone culture results.

\section{References}

1. Mandell GL. Osteomyelitis. in Mandell, douglas, and bennett's, principles and practice of infectious diseases G.L. Mandell, Bennett, J.E., Dolin, R., Editor. 2010; Churchill Livingstone: Phladelphia,PA, USA. . 14611462.

2. Murray PR. Enterobacteriaceae. in Medical microbiology. P.R. Murray, Editor. 2009; Mosby Elsevier: Philadelphia, PA. 301-314.

3. Tellez I, Chrysant GS, Omer I, Dismukes WE. Citrobacter diversus endocarditis. Am J Med Sci. 2000;320(6):408410.

4. Kleint W, Herwig H. Septic osteomyelitis caused by Citrobacter with a contribution to the problem of cortisone administration in bacterial infections. Arztl Wochensch. 1958;13(43):965-969.

5. Jansen RD, Meadow WL, Schwartz IK, Ogata ES. "Bacteriological bit": Citrobacter diversus osteomyelitis in a neonate. Clin Pediatr (Phila). 1981;20(12):791.

6. Lipsky BA, Hook EW, 3rd, Smith AA, Plorde JJ. Citrobacter infections in humans: experience at the Seattle Veterans Administration Medical Center and a review of the literature. Rev Infect Dis. 1980;2(5):746-760.

7. Drelichman V, Band JD. Bacteremias due to Citrobacter diversus and Citrobacter freundii. Incidence, risk factors, and clinical outcome. Arch Intern Med. 1985;145(10):1808-1810.

8. Wong SK, Wilhelmus KR. Infantile maxillary osteomy- 
elitis with cerebral abscess. J Pediatr Ophthalmol Strabismus. 1986;23(3):153-154.

9. Hartemann-Heurtier A, Senneville E. Diabetic foot osteomyelitis. Diabetes Metab. 2008;34(2):87-95.

10. Edmonds $M$. The treatment of diabetic foot infections: focus on ertapenem. Vasc Health Risk Manag. 2009;5:949-963.
11. Lipsky BA. Osteomyelitis of the foot in diabetic patients. Clin Infect Dis. 1997;25(6):1318-1326.

12. Newman LG, Waller J, Palestro CJ, Schwartz M, Klein MJ, Hermann G, Harrington E, et al. Unsuspected osteomyelitis in diabetic foot ulcers. Diagnosis and monitoring by leukocyte scanning with indium in 111 oxyquinoline. JAMA. 1991;266(9):1246-1251. 